\title{
Modeling Vowel Quantity Scales in Q Theory
}

\author{
Karee Garvin, Myriam Lapierre, Martha Schwarz, Sharon Inkelas \\ University of California, Berkeley
}

\section{Background: a four-point scale}

A growing body of research suggests that vowels vary in degree of strength. These strength differences are borne out in the degree to which these segments undergo or trigger phonological processes such as stress assignment or harmony. Traditionally, this variability has been accounted for through binary differences in phonological representations, such as presence vs. absence of a segment in the underlying representation, presence or absence of a phonological feature, or moraicity vs. non-moraicity of the relevant segment. For instance, in many languages, inserted vowels act invisible to stress assignment, often resulting in noncanonical stress patterns in an otherwise regular metrical system (Alderete 1999, Elfner 2016). This observation has given rise to grammatical constraints such as HEAD-DEP (Alderete 1999), which penalizes stress assignment to a segment that is absent from the input; or NON-FOOT(ə), which penalizes the assignment of metrical structure to a schwa vowel (Cohn \& McCarthy 1998). Distinctions in underlying status and moraic structure are an effective tool for capturing some, but not all, of the observed differences in vowel strength. In this paper, we provide evidence for a four-point scale of strength. Building on Schwarz et al. (2019), we argue that Q-Theoretic representations offer the necessary representational tool to capture this vowel strength scale as it is manifested in stress assignment and epenthesis patterns.

In Q Theory (Shih \& Inkelas 2019), a canonical short segment Q has three $q$ subsegments (q q q), roughly corresponding to onset, target, and offset release of an articulatory gesture within the framework of Gestural Coordination Theory (Gafos 2002). Segments may deviate from this canon by possessing more or fewer subsegments (Inkelas \& Shih 2017; Garvin et al. 2018). We argue that the four-point Q-Theoretic scale in (1), ranging from super-light (v) to phonologically long (vvvv) vowels, captures the observed range of scalar vowel behavior in ways previous models of phonological representations cannot.
(1)
Weakest vowels | (v)
(vv)
(vvv)
(vvvv) | Strongest vowels

While not all languages require all four points on the vowel strength scale, some do. We argue, on the basis of case studies from Panãra and Mohawk, that this scale is not reducible to presence or absence in underlying representation, nor to moraicity. We show that subsegmental representations are independent from, and compatible with, the well-motivated, but more limited distinctions that can be achieved in these ways.

This paper is organized as follows: Section 2 motivates a four-point scale of strength and applies this scale, in section 2.1, in case studies of Panãra and Mohawk. Section 2.2 addresses the relation between subsegmental and moraic structure. Section 3 formalizes the stressability scale in Q-Theoretic terms; Section 4 formalizes the epenthesis strength scale in Q-Theoretic terms; and Section 5 concludes.

\footnotetext{
${ }^{1}$ We thank audiences at UC Berkeley's Phonetics and Phonology Forum and the Annual Meeting on Phonology in Stony Brook for insightful feedback on earlier presentations of this work. We would also like to thank the Panãra community for allowing us to study their language, and Jesse Zymet for discussions on Mohawk epenthesis and stress assignment.
} 


\section{Evidence for a four-point scale}

Vowels of otherwise similar quality do not all behave alike in terms of strength. Sometimes, strength differences are attributed to underlying vs. epenthetic status; sometimes they are attributed to moraic weight. But neither dimension correlates sufficiently with the observed data. For example, it is not the case that epenthetic vowels are uniformly weak (e.g. unstressable, prone to deletion, short). Both epenthetic and lexical vowels can, and do, exhibit strength differences. For instance, Gafos (2002) and Hall (2006) demonstrate a behavior distinction among short inserted vowels (excrescent vs. epenthetic): In Scottish Gaelic (Hall 2006, Hammond et al. 2014) and Axininca Campa (McCarthy \& Prince 1993), inserted vowels can be longer than some underlying vowels. It is also not the case that (short) underlying vowels are uniformly strong; for example, see Smith (2017) on the varying degrees of strength demonstrated by harmony triggers in Rejang (ISO code rej) and Classical Manchu (ISO code mnc).

Even long vowels can vary in the degree of strength they exhibit. For instance, in Scottish Gaelic, both inserted and underlying vowels can be long, but differ in their ability to head a syllable (for discussion, see Hall 2006, Hammond et al. 2014). Given strength differences even within the categories that can be adduced by crossing the epenthesis with the moraicity dimension, these examples and others we will discuss show the need for a richer scale of segment strength.

Our proposal, the scale in (1), offers four choices for the representation of any given vowel, to which constraints governing epenthesis (faithfulness) and mora and stress assignment (markedness) can refer. This model allows strength differences among underlying and inserted vowels, and within monomoraic and bimoraic vowels as well, subject to scalar implications discussed below. For example, insertion of subsegmental units interacts with faithfulness constraints, making it more costly to insert a strong segment with more subsegments than a light segment with fewer subsegments. As such, the interaction between strength and faithfulness predicts that inserted segments are more likely to be light and super light segments, but also leaves open the possibility that strong segments may be inserted, given sufficient motivation within the grammar. These predictions are borne out in examples such as Axininca Campa, in which word minimality considerations lead to long vowel epenthesis (McCarthy \& Prince 1993).

In subsection 2.1, we explore in more detail the ability of the strength scale in (1) to capture strength differences among what are traditionally described as "short" (i.e., not bimoraic) vowels. In 2.2 we explore interactions between $\mathrm{Q}$ Theory and moraic structure.

2.1 Light, super-light, and full vowels Patterns of strength variability in inserted and underlying short vowels have proved problematic to represent in traditional models. Turkish (Bellik 2018) and Moroccan Arabic (Gafos 2002) share the distinction between (a) 'excrescent' vowels which, while reliably present, lack a distinct target and are shorter in duration; (b) more robust but arguably epenthetic vowels which inhabit a restricted inventory of targets; and (c) full, invariant, short (monomoraic) lexical vowels whose quality ranges over the full vowel inventory.

Additionally, underlying vowels may pattern with varying degrees of strength, such as examples like San Miguel el Grande Mixtec (MIG; ISO code mig). Zimmerman (2018) demonstrates that tonal patterns in MIG can be captured through different degrees of strength in underlying segments. In MIG, 'perturbing' morphemes consist of a final floating high tone $(\mathrm{H})$ that is realized on the following morpheme as $\mathrm{H}$ (as in 2a-b, d-e). However, for an exceptional set of morphemes, including /-ðe/, the H tone is only realized when the final TBU of the 'perturbing' morpheme is not $\mathrm{H}$ (as in 2c).

(2) Exceptional non-host for floating H-tone (McKendry 2013)
a. $/$ nut $\mathbf{1}^{(\mathrm{H}) /}$ 'bean' +
b. /jee ${ }^{(\mathrm{H}) /}$ 'eat' $\quad+$
/-ðе/ '3.MHON' $\rightarrow$
c. / $/ \mathrm{áá}^{(\mathrm{H}) /} \quad$ 'EMPH' +
-ðе/ '3.MHON' [nutfiðé]
[jeeðé]
/-ðe/ '3.MHON' $\rightarrow$ [bááðe]
$\rightarrow \quad$ [báátí]
d. $/ \beta$ áá $^{(\mathrm{H}) /}$ 'EMPH' + /-tid/ '3.ANIM'
/-t firí/ 'skunk'
[Sinít Jî́í]
(McK 2013:92)
(McK 2013:104)
(McK 2013:92)
(McK 2013:92)
(McK 2013:85)

As shown by example (2d), a simple ban on neighboring H-tones cannot account for the pattern, as (2d) does surface with $\mathrm{H}$ tone while (2c) does not. Thus, Zimmerman (2018) concludes that the exceptionality in examples such as (2c), can be accounted for through different strengths of activation between underlying segments, where the underlying representation is too weak to host the floating tone. The vowels in these 
examples are all underlying, and therefore, binary distinctions of strength are insufficient for capturing within-category differences in segment strength.

We present, below, two case studies demonstrating the necessity for a Q-Theoretic model of strength: Panãra (Section 2.1.1) and Mohawk (Section 2.1.2).

2.1.1 A case study of Panãra As argued in previous work (Schwarz et al. 2019), the Jê language Panãra (ISO code: kre) provides a clear example of a language where a four-way contrast in vowel strength offers the necessary representational machinery to capture surface forms and phonological alternations such as stress assignment. Panãra exhibits a contrast in vowel length for 13 vowel qualities (3) and additionally exhibits both epenthetic and excrescent vowels ${ }^{2}$ (Lapierre 2019).
a. $/ \mathrm{pa} /$
[pa]
'arm'
c. /sõş/
[sõ. 's $\mathrm{s}]$
'greedy'
b. /pa:/
[pa:]
'foot'
d. /sõ:ş/
[sõ:. 's $\mathrm{s}$ ]
'longing(for someone)'

Stress in Panãra always falls within a two-syllable window at the right edge of the word, meaning that it always falls on the ultimate (3-4) or the penultimate syllable (5). The examples in (5) show that, when stress falls on the penultimate syllable of the phonological word, the stressed vowel is always lengthened, and the last vowel of the word is generally $[i]^{3}$.
a. /soti/
[so.'ti]
'fox'
b. /kukrel
[ku. 'kre]
'house'
d. /kotiko/
e. /prtiti/
[ko.ti.'ko]
'village name'
c. /nãsu//
[nã.'su]
'deer'
f. /tupajurõ/
[pr.ti.'ti]
'anteater'
a. /tep/
['tc:.pi]
'fish'
e. /popot/
[tu.pa.ju..' rõ]
'village name'
b./sot/ ['so:.ti] 'stuff'
c. $/$ pro/ ['pr:..i] 'tree'
f. /prr-kjat/
[po.'po:ti] 'round'
d. /aprẽp/
[a.'prẽ̃.pi] 'picture, spirit'
g. /tcp-akrit/
[pr.ri.'kja:.ti] 'tree stump'
[te.pa.'kri:ti] 'whale'

Crucially, word-final [i] is not observed when the relevant root attaches to the left of a morpheme beginning with another oral consonant, as can be seen by comparing (5a) and (5b) to (6a) and (6b), respectively. By contrast, stressed word-final [i] does not disappear under morpheme concatenation, as shown in (7).
a. /tep-pã:/ [tєp.'pã:] 'small fish'
b. /sot-pjo/
[sop.'pjo]
'nothing, lit. no things'
a. /soti-pã:/ [so.ti.'pã:] 'small fox'
b./prtiti-pã:/
[pr.ti.ti.'pã:]
'small anteater'

Together, these facts suggest that the word-final [i] observed in examples (5) is an epenthetic vowel. As argued in Lapierre (2019), this epenthetic [i] arises as a way to resolve a ban on word-final oral consonants. Indeed, neither obstruent nor approximant oral consonants are observed in word-final position in Panãra. Only nasal codas are observed in this position, as in (8).
a. /kan/
[kan]
'basket'
b. /twatũn/
[twa.' 'tũm]
'elderly woman'

Short inserted vowels are also observed between two consonants in an onset cluster composed of an obstruent followed by an approximant. Like in the case of word-final [i], these vowels are never stressed, and their quality is entirely dependent on the quality of the surrounding vowels and consonants, as can be observed in (9). Unlike word-final [i], however, native speaker intuitions suggest that these short vowels do not constitute a syllable head.
a. $/ \mathrm{krr} /$
$\left[\mathrm{k}^{\mathrm{r} \gamma}\right]$
'thigh'
b. /swa/
$\left[\mathrm{s}^{\mathrm{u}} \mathrm{wa}\right]$
'tooth'

\footnotetext{
2 For a full overview of Panãra phonology, see Lapierre (2019).

3 When the relevant morpheme ends in [op] or [op], the word-final epenthetic vowel is realized as [u]], and some morphemes select for word-final [a] instead of word-final [i].
} 
The facts summarized here suggest a four-point strength scale for vowels in Panãra, and we offer the following Q-Theoretic representations to capture these facts. Super-light vowels arise between onset consonants, and their quality is entirely determined by adjacent consonants and vowels (10a). They are represented as one $q$ subsegment (v). Light vowels arise as a way to resolve a ban on word-final oral codas, and they crucially cannot bear stress, despite otherwise systematic final stress in the language (10b). Their default quality is [i], thus representing a subset of the full vowel inventory. Accordingly, they are represented with two subsegments (vv). Full (vvv) vowels (10c) and phonologically long (vvvv) vowels (10d) differ from one another in length and from super-light and light vowels in their ability to bear stress, and they represent the full range of quality contrasts, namely 15 short vowels and 13 long vowels.
a. $/ \mathrm{kr \gamma} / \quad\left[\mathrm{k}^{\gamma} \mathrm{r \gamma}\right]$
b. $/ \mathrm{t} \varepsilon \mathrm{p} / \quad$ [te.pi]
c. $/ \mathrm{pa} / \quad$ [pa]
$(\mathrm{k} \mathrm{kk})(\gamma)(\mathrm{r} r)(\gamma \gamma \gamma)$
$(\mathrm{t} \mathrm{t} t)(\varepsilon \varepsilon \varepsilon)(\mathrm{p} \mathrm{p} \mathrm{p})(\mathbf{i} \mathbf{i})$
'thigh'
(p p p) (a a a)
d. $/ \mathrm{pa} / \quad[\mathrm{pa}:]$
(p p p) (a a a a)
'fish'
'arm'
'foot'

In addition to the vowel strength distinctions above, rime weight in Panãra bears on the interaction between segment strength and weight. While the full pattern of word-initial [i] epenthesis is quite complex and a full description of the system is beyond the scope of this paper, word-minimality facts show that VC rimes are heavier than both $\mathrm{V}$ : and $\mathrm{V}$ rimes. The crucial pattern is exemplified below in (11), where monosyllabic words with a $\mathrm{V}$ : and $\mathrm{V}$ rimes can be augmented by a word-initial [i] vowel epenthesis process, (11a-b), but monosyllabic words with a VC rime cannot be realized with the same word-initial epenthetic [i] $(10 c-d)^{4}$.
a. $/ \mathrm{pa} /[\mathrm{pa} \sim \mathrm{i} . \mathrm{pa}]$ 'arm'
c. $/ \mathrm{kan} /$
b. /pa:/
[pa i.'pa:] 'foot'
d. $/ \mathrm{trn} /$
[kan], *[i.'kan] 'basket'
[try], *[i.'try] 'raw'

An analysis of this pattern in terms of mora assignment constraints which refer to points on the four-point strength scale in (1) is presented below, in Section 2.2.

2.1.2 A case study of Mohawk Mohawk (Iroquoian, ISO code: moh) exhibits a pattern of stress assignment and stress shifting similar to that described above for Panãra. The data from Mohawk also crucially exemplify the need for a four-point strength scale for vowels. The pattern described here directly follows its discussion in Adler \& Zymet (in press), and the primary data are from Michelson $(1988,1989)$. In Mohawk, stress generally falls on the penultimate syllable of a word, as shown in (12). In cases where the stressed penultimate syllable is open, the vowel is lengthened (12c-d). Note that, unlike in Panãra, vowel length is not contrastive in Mohawk.
a. /k-atirut-ha?/
[ka.ti.' rut.ha?]
b. /k-ohar-ha?/
[ko.'har.ha?]
c. /k-haratat-s/
[kha.'ra:.tats]
d. /wak-aru?tat-u/
[wa.ka.ru?.'ta:.tu]
'1A-pull-HAB'
'1A-attach- HAB'
'1A-lift- HAB'
'1P-blow-STAT'

(Mson 1988:53)

(Mson 1988:53)

(Mson 1988:53)

(Mson 1988:59)

Furthermore, an epenthetic vowel [e] arises in two phonotactic contexts. This vowel is inserted between the first two consonants in a CCC sequence (13), as well as inside of a CC sequence consisting of an oral obstruent and a sonorant (14).
a. /s-k-ahkt-s/
b. /wak-nyak-s/
['skah.kets]
[wa.' ken.yaks]
c. /te-k-ahsutr-ha?/
[te.kah.su.'ter.ha?]
'1 P-get.married- HAB'
'DU-1A-splice- HAB'
'ITER-1A-go.back- HAB'

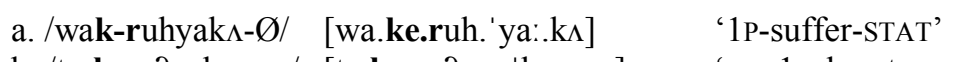
b. /te-k-ra?nekar-us/ [te.ke.ra?.ne.'ka:.rus]
'DU-1A-burst- HAB'

(Mson 1988:135)

(Mson 1988:135)

(Mson 1988:142)

(Mson 1988:134)

(Mson 1989:41)

\footnotetext{
${ }^{4}$ For a full review of word-minimality and word-initial [i] epenthesis patterns in Panãra, see Lapierre (2019).
} 
Vowel epenthesis interacts with stress when it occurs in the penultimate syllable of the word. Adler \& Zymet (in press) state that if [e] occupies a closed penult, the penult is stressed (14), and if [e] occupies an open penult, the antepenult is stressed (15). Note that if the antepenult is open, the stressed vowel is not lengthened.
a. /wak-nyak-s/
[wa.' ken.yaks]
b. /te-k-ahsutr-ha?/
[te.kah.su.'ter.ha?]
c. /k-rho-s/
['ker.hos]
d. /ak-tshe-?/
[a.' ket.she?]
a. /te-k-rik-s/
['te.ke.riks]
b. / $/$-k-r- $\Lambda$ ?/
[' $\Lambda . k e . r \Lambda$ ?
c. /W-akra-s/
['wa.ke.ras]
d. /te- $\Lambda$-k-ahsutr- $\Lambda$ ?/ [t $\quad$.kah. 'su.te.r $\Lambda$ ?]

'1P-get.married- HAB'
'DU-1A-splice- HAB'
'1A-coat, spread- HAB'
'1P-container-NSF'
'DU-1A-put together- HAB'
'FUT-1A-put.in-PUNC'
'NA-smell- HAB'
'DU-FUT-1A-splice- HAB'

(Mson 1988:134)

(Mson 1988:142)

(Mson 1988:137)

(Mson 1989:42)

(Mson 1988:133)

(Mson 1988:134)

(Mson 1988:141)

(Mson 1988:142)

Another clear pattern that arises from the grouping of the data above, however, is that those epenthetic vowels that arise in CCC sequences may bear stress (15), while those that arise in obstruent-sonorant sequences cannot (16). While Adler \& Zymet (in press) work within a framework that requires them to treat all epenthetic vowels in the same way, we note that the vowels inserted in these two different phonotactic contexts in fact exhibit different sets of phonological behavior with respect to stress assignment. We appeal to Q Theory to offer an alternative analysis of these facts, proposing that the [e] vowel observed in (13) and (15) has two subsegments (vv), while the that in (14) and (16) has one (v). This distinction is consistent with the typology of inserted vowels presented by Hall (2006) and with the categorization of the pattern in (14) and (16) as an instance of Dorsey's Law (Steriade 1990), identical to the pattern described above for excrescent (v) in Panãra (see (9) and (10a)).

In Mohawk, super-light vowels [e] arise inside of oral obstruent-sonorant sequences, and they crucially cannot bear stress despite otherwise systematic penultimate stress in the language (17a). Light vowels [e] arise as a way to resolve a ban on CCC sequences (17b). Full vowels are contrastive (17c), and long vowels arise when a stressed penultimate vowel is lengthened because it occurs in an open syllable (17d).
a. /w-akra-s/
['wa.k(e).ras]
b. /te-k-ahsutr-ha?/
[te.kah.su.'t(e e)r.ha?]
'NA -smell- HAB'
c. /k-atirut-ha?/
[ka.ti.' r(u u u)t.ha?]
'DU -1A-splice- HAB'
' $1 \mathrm{~A}$-pull- HAB'
d. /wak-ruhyaks-Ø/

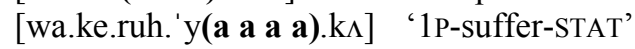

(Mson 1988:141)

(Mson 1988:142)

(Mson 1988:53)

(Mson 1988:134)

An interesting observation that arises from the comparison of the Mohawk and the Panãra data discussed in this section is that the threshold at which vowels can and cannot bear stress is language-dependent. In Panãra, only vowels with at least three subsegements (vvv) may bear stress, whereas in Mohawk, only two (vv) subsegments are required for a vowel to bear stress. This observation is predicted and discussed in (Schwarz et al. 2019). While the number of subsegments required for a vowel to be able to bear stress is dependent on idiosyncratic facts about that language's grammar, what is systematic is the observation that if a vowel with, for example, two subsegments can bear stress, this necessarily implies that a vowel with more than two subsegments will also be able to bear stress (see Sections $2.2 \& 3$ ).

2.2 $Q$ Theory and moraic scales Analyzing moraic structure and strength as independent allows for more fine-grained analysis of both length and weight. Both Panãra and Mohawk demonstrate not only a need for a four-point strength scale (1), but also the necessity for strength and weight to be independent. For instance, in the Panãra data in Section 2.2, long vowels do not attract stress, but light and super-light vowels cannot be stressed. This suggests that long vowels are not metrically heavier than other full vowels in the language (Schwarz et al. 2019, Lapierre 2019). Similarly, in Mohawk, super-light vowels cannot be stressed but light vowels can be. Furthermore, as Ryan (2018) argues, a number of quantity-sensitive stress systems exhibit a weight scale in which $\mathrm{VVC}>>\mathrm{VV}>\mathrm{VC}>>\mathrm{V}$. This four-point scale cannot be captured using only the binary distinction between $\mu$ and $\mu \mu$, even when coercion (Morén 2000) is invoked. Ryan (2018) argues persuasively that the mora scale has to be supplemented with other prominence scales.

One such example where a binary strength scale is insufficient comes from Nanti stress assignment. Nanti stress assignment illustrates the claim that strength and weight are independent, as syllable weight is 
dependent on vowel length, vowel quality, as well as presence or absence of a coda consonant (Crowhurst \& Michael 2005:66). This is formalized in the scale in (18).

$$
\begin{aligned}
& \text { CaaN, CaVN } \gg>\text { CeeN CeiN, CooN, CoiN, CuiiN } \gg>\text { CiiN, Caa, CaV } \gg>\text { Cee, Cei, Coo, Coi, Cuii } \\
& >\mathrm{Cii} \gg \mathrm{CaN} \gg \mathrm{CeN}, \mathrm{CoN}, \mathrm{CuiN} \gg \mathrm{CiN} \gg \mathrm{Ca} \gg>\mathrm{Ce}, \mathrm{Co}, \mathrm{Cui} \gg \mathrm{Ci}
\end{aligned}
$$

The Nanti strength scale in (18) shows that both vowel strength and moraic structure must operate in tandem to capture the hierarchy of stress sensitivity. As Ryan (2018) has concluded, moraic structure must be supplemented with additional machinery in order to capture the full range of strength and stressability.

Examples such as Nanti, Scottish Gaelic and Axininca Campa highlight the need for more granularity in our representational tools. In the Q-Theoretic representations proposed here, long vowels needn't be tied by default to a bimoraic structure. Instead, moraic structure can work together with vowel strength, such that long vowels may be represented as monomoraic or bimoraic depending on weight sensitivity patterns. In the following subsection, we outline the interaction between Q-Theoretic representations of strength and moraic structure to illustrate how these representations can be leveraged to capture the full range of attested patterns of strength and weight.

2.2.1 Subsegments are skeletal units Q Theory can represent the difference between full and long vowels in terms of subsegmental quantity: A full vowel has three subsegments, and a long vowel has four, as in (19). Note that the second subsegment, $\mathrm{v}^{2}$, is repeated, to formalize the observation that it is the gestural plateau that is lengthened (see Gafos 2002).

$$
\begin{array}{ll}
\text { a. Full vowel: } & \left(\mathrm{v}^{1} \mathrm{v}^{2} \mathrm{v}^{3}\right) \\
\text { b. Long vowel: } & \left(\mathrm{v}^{1} \mathrm{v}^{2} \mathrm{v}^{2} \mathrm{v}^{3}\right)
\end{array}
$$

This length distinction co-exists with, but does not replace the use of moras, which have been used since Hyman (1985) (see also Zec 1988 and Hayes 1989) to distinguish a short (one mora) from a long (two mora) vowel or even, in some cases, a three-mora vowel. In Q Theory, subsegments are skeletal units; subsegmentally complex representations replace the "V" in traditional moraic representations, as in (20).
a. $\mu$
V
b. $\begin{gathered}\mu \mu \\ V \\ V\end{gathered}$

The moraic weight scale and the Q-Theoretic subsegmental strength scale are independent scales which co-exist in the grammar. Both scales can be referred to by constraints on syllable structure, stress, and moras (see Section 4). Subsegments and moras can be aligned via grammatical constraints, building on existing literature in which moraic structure emerges from segmental structure. For example, the assumption of Hyman (1985) and Hayes (1989) that all vowels are associated with at least one mora can be treated as a mora projection constraint. The constraint in (21), requiring segments above a certain minimum sonority threshold to be dominated by at least one mora, at a minimum requires each vowel to be moraic. If the sonority threshold is low, then it also achieves the 'Weight-by-Position' effect that, in some languages and subject to language-particular sonority thresholds, coda consonants also contribute a mora to the syllable in which they are contained (see e.g., Zec 1998, Morén 2000, Ryan 2018, among others).

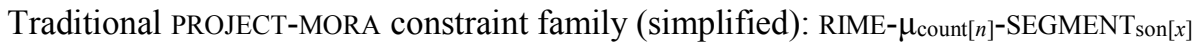

Each rime segment of sonority threshold $\geqq x$ must be dominated by at least one mora.

Example members of the constraint family:

a. Each rime vowel must be dominated by at least one mora

b. Each rime sonorant consonant must be dominated by at least one mora

c. Each rime consonant must be dominated by at least one mora (Weight-by-Position; Hayes 1989) 
In standard moraic theory (e.g., Hayes 1989), lexically long vowels require two moras in their underlying representation, as length is not predictable from anything else in the representation. A family of markedness constraints regulates the distribution of moras to prevent unwanted mora sharing and syllables that would exceed two moras. Constraint (22a) is needed to rule out mora sharing in languages, and constraint (22b) prohibits overly heavy syllables.

(22) Mora minimization constraints (based on previous literature)

a. Penalize structures in which one mora is associated with more than one segment

b. Penalize syllables with more than two moras

These standard constraints, rooted in syllable position, segment count, and segment sonority, correctly generate the patterns observed by Hyman (1985), Hayes (1989), Zec (1988) and many others, that in some languages, both $\mathrm{CV}$ : and $\mathrm{CVC}$ are bimoraic, and that in other languages both $\mathrm{CVC}$ and $\mathrm{CV}$ are monomoraic. However, as the Panãra and Mohawk data above illustrate, not all moraic scales are of these two types. By replacing the notion of 'segment' in the mora projection constraint family with the notion of 'subsegment,' a new Q-Theoretic PROJECT-MORA constraint family emerges, with the capacity to capture a wider variety of attested mora projection patterns. Example (23) provides a schema for mora projection that is sensitive to both subsegmental sonority and subsegmental quantity.

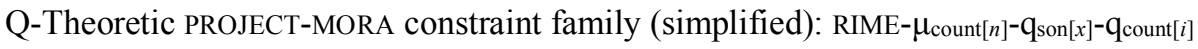

Each rime sequence of $i$ or more subsegments, all of which are of sonority threshold $\geqq x$, must be dominated by at least $n$ moras

Note that in Q Theory, underlying vowel length can be represented subsegmentally, allowing even the moraic structure of long vowels to emerge from the grammar (24c) rather than be present in the underlying representation.

(24) Q-Theoretic PROJECT-MORA constraint family (examples)

a. $\quad$ RIME- $\mu-\mathrm{v}-[i]$ : Each rime sequence of $i$ or more $\mathrm{v}$ subsegments must be dominated by at least one mora

b. RIME- $\mu-c-[j]$ : Each rime sequence of $j$ or more c subsegments must be dominated by at least one mora

c. RIME- $\mu \mu-\mathrm{V}-[k]$ : Each rime sequence of $k$ or more v subsegments must be dominated by at least two moras

The above constraints can generate the two well-known systems (e.g, Zec 1988) in which CV: >> CVC, CV (25), and in which CV:, CVC $>$ CV (26).

$\mathrm{CV}:>\mathrm{CVC}, \mathrm{CV}$

$\mathrm{C}(\mathrm{vvvv}) \gg \mathrm{C}(\mathrm{vvv})(\mathrm{ccc}), \mathrm{C}(\mathrm{vvv})$

a. RIME- $\mu$-v-count[3]: Each rime segment composed of 3 or more $v$ subsegments must be dominated by at least one mora

b. RIME- $\mu \mu$-v-count[4]: Each rime segment which is vocalic and has 4 subsegments must be dominated by at least two moras

$\mathrm{CV}:, \mathrm{CVC} \gg \mathrm{CV}$

$\mathrm{C}(\mathrm{vvvv}), \mathrm{C}(\mathrm{vvv})(\mathrm{ccc})>>\mathrm{C}(\mathrm{vvv})$

a. RIME- $\mu$-q-count[3]: Each rime segment composed of $i$ or more subsegments must be dominated by at least one mora

b. RIME- $\mu \mu$-v-count[4]: Each rime segment which is vocalic and has 4 subsegments must be dominated by at least two moras

But these subsegment-based constraints, unlike the purely segment-based constraints in (21-22), can also generate additional patterns which have been difficult to capture using only full segments and moras, including those represented in the case studies above. In Panãra, for example, we saw evidence for a scale in 
which CVC >>CV:, CV. The bimoraic word minimality condition shows that codas make a syllable heavy, but long vowels do not. This generalization is consistent with standard moraic theory in which moras are projected from $\mathrm{V}$ and $\mathrm{C}$ skeletal units. In Q Theory, they can be captured by allowing mora projection to be sensitive to subsegmental quantity and quality. The subsegmental constraints in (25-26) can also capture the generalization observed in Panãra that epenthetic vowels may not bear stress.

In Panãra, to qualify as bimoraic, a syllable must have a rime with at least 6 subsegments, contributed by a full or long vowel plus a coda consonant. A (vv) light vowel is not sufficient to project a mora on its own, and a long (vvvv) vowel is not sufficient to project two moras on its own. These generalizations can be modeled by constraints in the family introduced above in (25-26), as in (27).

a. RIME- $\mu$-v-count[3]: If (vvv) or (vvvv), project a mora

b. RIME- $\mu-\mathrm{c}-\operatorname{count}[3]$ : If (ccc) or (cccc) in the rime, project a mora

Because long (vvvv) vowels project one but not two moras in Panãra (see Tableau 1), Q Theory can generate the observed Panãra weight scale, namely CVC >>CV:, CV (see Tableau 2).

\begin{tabular}{|c|c|c|c|c|c|}
\hline & $/ \mathrm{C}(\mathrm{vvvv}) /$ & RIME- $\mu$-v-count[3] & RIME- $\mu$-c-count[3] & ${ }^{*} \mu$ & $H$ \\
\hline & & 2.0 & 2.0 & 1.0 & \\
\hline $\mathrm{a}$ & $\mathrm{C}(\mathrm{vvvv})$ & -1 & 0 & 0 & -2 \\
\hline$\varpi \mathrm{b}$. & $\mathrm{C}(\mathrm{vvvv}) \mu$ & 0 & 0 & -1 & -1 \\
\hline c. & $\mathrm{C}(\mathrm{vvvv}) \mu \mu$ & 0 & 0 & -2 & -2 \\
\hline
\end{tabular}

Tableau 1: Long vowels project a single mora

\begin{tabular}{|c|c|c|c|c|c|}
\hline & $/ \mathrm{C}(\mathrm{vvv})(\mathrm{ccc}) /$ & RIME- $\mu$-v-count[3] & RIME- $\mu$-c-count[3] & ${ }^{*} \mu$ & $H$ \\
\hline & & 2.0 & 2.0 & 1.0 & \\
\hline $\mathrm{a}$ & $\mathrm{C}(\mathrm{vvv})(\mathrm{ccc})$ & -1 & -1 & 0 & -4 \\
\hline b. & $\mathrm{C}(\mathrm{vvv})(\mathrm{ccc}) \mu$ & -1 & 0 & -1 & -3 \\
\hline c. & $\mathrm{C}(\mathrm{vvv}) \mu(\mathrm{ccc})$ & 0 & -1 & -1 & -3 \\
\hline m. & $\mathrm{C}(\mathrm{vvv}) \mu(\mathrm{ccc}) \mu$ & 0 & 0 & -2 & -2 \\
\hline
\end{tabular}

Tableau 2: Coda consonants project a single mora

A slight refinement of the constraints in (27) produces the stress system seen above in Mohawk, in which (v) is not stressable, but (vv), (vvv), and (vvvv) are all stressable, suggesting that all vowels with more than one subsegment are moraic. As was shown, coda consonants are also moraic. These observations can be modeled as in (28).

a. RIME- $\mu$-v-count[2]: If (vv), (vvv) or (vvvv), project a mora

b. RIME- $\mu$-c-count[3]: If (ccc) is in the rime, project a mora

In terms of mora count alone, $\mathrm{Q}$ Theory predicts that a syllable containing $\left(\mathrm{v}^{\mathrm{n}+1}\right)$ will never be lighter than $\left(\mathrm{v}^{\mathrm{n}}\right)$, all else being equal. Q Theory predicts that the moraicity of $(\mathrm{vvv})(\mathrm{ccc})$ and $(\mathrm{vvvv})$ can vary across languages. In some languages, a $(\mathrm{vvv})(\mathrm{ccc})$ syllable rime is bimoraic and a (vvvv) syllable rime is monomoraic; in others, both such rimes are bimoraic; and in others yet, only (vvvv) is bimoraic. Of course, mora count is not the only factor that contributes to stressability, as discussed in the next section.

\section{Formalizing the stressability scale in Q-Theoretic terms}

Ryan (2018) suggests adding specific markedness constraints, such as V:-STRESS, to capture weight distinctions that elude moraic characterizations. Q Theory offers a more uniform alternative; its greater granularity offers inherent, scalar distinctions, measured in subsegments, of the vowel and total durations of 
syllable rimes. The $\mathrm{V}: \mathrm{C}>\mathrm{VV}>\mathrm{VC}>\mathrm{V}$ weight scale (29) emerges naturally from subsegmental quantity and the constraints in (30):

Rime weight scale with subsegments: $(\mathrm{vvvv})(\mathrm{ccc})>(\mathrm{vvvv})>(\mathrm{vvv})(\mathrm{ccc})>(\mathrm{vvv})$

Schwarz et al. (2019) propose a set of stringent Q-Theoretic stress constraints which ensure that, all else being equal, a syllable headed by more $\mathrm{v}$ subsegments is a better stress target than one headed by fewer:

*STRESS/v: Assign one violation for every vowel with one subsegment that bears stress

* STRESS/v, vv: Assign one violation for every vowel with one or two subsegments that bears stress

* STRESS/v, vv, vvv: Assign one violation for every vowel with one, two, or three subsegments that bears stress

* STRESS/v, vv, vvv, vvvv: Assign one violation for every vowel with one, two, three, or four subsegments that bears stress

Paired with the mora projection constraints in (23-26), these constraints can derive the scale observed by Ryan (2018). Weighted appropriately, the mora projection constraints in (23-26) can ensure that a $(\mathrm{vvv})(\mathrm{ccc})$ syllable rime is heavier, and thus a better stress target, than a (vvv) rime. The constraints in (30) ensure, all else equal, that a syllable containing (vvvv) is a better stress target than one containing (vvv). Whether (vvvv)(ccc) is trimoraic, or simply a better stress target than (vvv)(ccc), depends on one's stance regarding trimoraic syllables, a question on which we do not take a position in this paper.

In addition to stressability, the four-point scale produces families of other markedness constraints as well. All else being equal, the ability to head a syllable, for example, is more marked for weaker vowels than for stronger vowels. The ability to exhibit contrast is also more marked for weaker vowels than stronger vowels. Panãra's inventory above exemplifies this: while there are 15 and 13 different qualities for full (vvv) and long (vvvv) vowels respectively, light (vv) vowels may only be [i, a, u] , and super-light (v) vowels have no inherent quality; rather, their quality is determined entirely by the surroundings segments.

\section{Formalizing the epenthesis strength scale in Q-Theoretic terms}

As seen in the Panãra, Mohawk, Axininca Campa, and Nanti examples above, inserted vowels can display a range of strengths. Yet cross-linguistically, inserted vowels tend to be weaker than canonical vowels, and deficient in properties like inherent quality and stressability. Within the framework proposed in this paper, epenthesis tends to correlate with weakness on the Q-Theoretic scale. In this section, we offer an explanation for this typological tendency.

First, we use the four-point representational strength scale presented and motivated above to generate families of stringent faithfulness and markedness constraints, using de Lacy's (2004) machinery. The families of faithfulness constraints DEP and MAX are given in (31).

$$
\begin{aligned}
& \text { a. DEP (vvvv), DEP (vvvv,vvv), DEP (vvvv,vvv,vv), DEP(vvvv,vvv,vv,v) } \\
& \text { b. MAX (vvvv), MAX (vvvv,vvv), MAX(vvvv,vvv,vv), MAX(vvvv,vvv,vv,v) }
\end{aligned}
$$

When used to evaluate a candidate, an inserted (vvvv) vowel will violate all four of the constraints in the DEP family, while an inserted (v) vowel will only violate one. Thus, epenthesizing a strong vowel (i.e. a vowel with more subsegments) will always incur more violations than epenthesizing a weaker vowel with fewer subsegments. Similarly, the family of MAX constraints ensures that deleting a stronger vowel will always violate more MAX constraints than deleting a weaker vowel. Weaker vowels are also expected to be more susceptible to assimilation than stronger vowels, which may be modelled with IDENT-q constraints that penalize processes that change the identity of a greater number of subsegment qs than processes that change the identity of fewer subsegment qs (Shih \& Inkelas 2019).

As previewed above, these faithfulness constraints also interact with markedness constraints, which may require insertion, deletion, or identity change of vowels with greater degrees of strength. In the rest of this section, we show how these interactions may predict the two distinct vowel insertion processes of Panãra described above in Section 2.2. We situate the analyses in Harmonic Grammar (Legendre et al. 1990). All constraint weights were automatically generated using OT-Help (Staubs et al. 2010). 
In the first Panãra vowel insertion process that we examine, super-light (v) vowels arise between onset consonants $\left(/ \mathrm{kr \gamma} /\right.$ 'thigh' $\rightarrow\left[\mathrm{k}^{\gamma} \mathrm{r} \gamma\right]$, as in (9)). Tableau 3 considers candidates with insertion of vowels with one, two, three, and four subsegments. The DEP constraints interact with an alignment constraint that penalizes two consecutive consonants with high degrees of gestural overlap (see Gafos 2002). Crucially, this alignment constraint is distinct from *COMPLEXONSET because it does not require that the onset be broken up into two syllables. As we will return to later, the inserted vowel does not head a syllable.

\begin{tabular}{|r|c|c|c|c|c|c|c|}
\hline & $/ \mathrm{kr} \gamma /$ & ALIGNCC & DEP(vvvvv) & DEP(vvvv,vvv) & DEP(vvvv,vvv,vv,v) & DEP(vvvv,vvv,vv,v) & $H$ \\
\hline & & 2.0 & 1.0 & 1.0 & 1.0 & 1.0 & 0 \\
\hline $\mathrm{a}$. & $\mathrm{kr} \gamma$ & -1 & 0 & 0 & 0 & -1 & -2 \\
\hline $\mathrm{b}$ b. & $\mathrm{k}(\gamma) \mathrm{r} \gamma$ & 0 & 0 & 0 & 0 & -1 & -1 \\
\hline $\mathrm{c}$. & $\mathrm{k}(\gamma \gamma) \mathrm{r} \gamma$ & 0 & 0 & 0 & -1 & -1 & -2 \\
\hline $\mathrm{d}$. & $\mathrm{k}(\gamma \gamma \gamma) \mathrm{r} \gamma$ & 0 & 0 & -1 & -1 & -1 & -4 \\
\hline $\mathrm{e}$. & $\mathrm{k}(\gamma \gamma \gamma \gamma) \mathrm{r} \gamma$ & 0 & -1 & -1 & -1 & & -1 \\
\hline
\end{tabular}

Tableau 3: Insertion of super-light (v) vowels in Panãra

In this analysis, ALIGN-CC is weighted highly enough to make the insertion of a vowel more optimal than the fully faithful form. However, ALIGN-CC is only weighted highly enough to cause the insertion of the weakest possible vowel, as the stringent DEP constraints ensure that nothing stronger than (v) is epenthesized.

In Panãra's second vowel insertion process, a light vowel (vv) arises word-finally to avoid word-final oral consonants. In addition to the cross-linguistically motivated *ORALCODA constraint, we utilize the constraint ${ }^{*} \mathrm{v}$ :

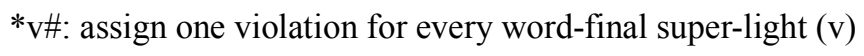

While * $\mathrm{v} \#$ might be motivated by the optimality of perceptually salient material word-finally, we use it primarily as a proxy constraint to stand in for broader syllable structure requirements of Panãra. In Panãra, the vowel inserted between segments in a complex onset are the result of low gestural overlap between the different articulatory gestures needed to produce the obstruent and following sonorant of the cluster. They cannot occupy the nucleus of a syllable (Lapierre 2019). The vowel inserted word-finally to avoid oral codas, however, crucially does need to change the syllable structure in order to produce a viable candidate that would allow the word-final consonant to resyllabify from a coda into an onset. Therefore, the grammar must insert a vowel that can head a syllable, of which a light (vv) vowel incurs the fewest DEP family violations. ${ }^{5}$

\begin{tabular}{|c|c|c|c|c|c|c|c|c|}
\hline & $/ \mathrm{tep} /$ & $*$ CODA & $*_{\mathrm{v}} \#$ & $\operatorname{DEP}(V V V V V)$ & DEP(VVVV,vVv) & DEP(vVVv,vVv,vv,v) & DEP(vvVv,vvV,vv,v) & $H$ \\
\hline & & 3.0 & 2.0 & 1.0 & 1.0 & 1.0 & 1.0 & \\
\hline a. & tep & -1 & 0 & 0 & 0 & 0 & 0 & -3 \\
\hline b. & $\operatorname{t\varepsilon p}(\mathrm{i})$ & 0 & -1 & 0 & 0 & 0 & -1 & -3 \\
\hline $\cos ^{\circ}$ & $\operatorname{tep}(\mathrm{i} \mathrm{i})$ & 0 & 0 & 0 & 0 & -1 & -1 & -2 \\
\hline d. & tep(i i i) & 0 & 0 & 0 & -1 & -1 & -1 & -3 \\
\hline e. & 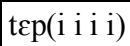 & 0 & 0 & -1 & -1 & -1 & -1 & -4 \\
\hline
\end{tabular}

Tableau 4: Insertion of light (vv) vowels in Panãra

In Sections 3 and 4 we have thus shown how producing markedness and faithfulness constraints based on the strength scale can explain both the typological tendency for the association between weak vowels and epenthesis, as well as the reasons for deviation from this tendency. The ability to capture two types of weak,

\footnotetext{
${ }^{5}$ Alternatively, we could achieve the same analysis with a constraint against inserting a vowel that cannot head a syllable in instances where syllable structure needs to be altered. This would serve the same purpose of making (vv) insertion more optimal than (v) insertion in word-final contexts but not in complex onset contexts.
} 
inserted vowels also brings the articulatory phonology distinction of excrescent vs. epenthetic vowels (Gafos 2002) straightforwardly into Optimality-Theoretic analyses.

\section{Conclusion}

In this paper, we have applied the four-level strength scale of vowels motivated in Schwarz et al. (2019) to explain the behaviors of different types of vowels that cannot be fully captured by the binary distinctions between underlying vs. inserted and between monomoraic vs. bimoraic. In this proposal, subsegments exist alongside moras, and are able to explain the strength scale observed by Ryan (2018), as well as vowelstrength sensitive patterns like that of Panãra, which aren't consistent with the predictions made a simple moraic system. The separation of strength from status as inserted vs. underlying allows for the full range of strengths across each type: Both inserted vowels and underlying vowels may range in quality from small and weak to full and strong. This is desirable because, as shown above, languages do show insertion of vowels of a wide range of strengths. Yet it is also true that cross-linguistically, inserted vowels tend to be weaker. We showed in Sections 3 and 4 that the families of constraints produced directly from the representations offer an explanation for these tendencies. Faithfulness constraints ensure that, all else being equal, inserted vowels will be weak. Only the interaction with markedness constraints will cause deviation from the typological norm. Thus, Q-Theoretic representations of vowel strength offer a unified framework for capturing the full range of attested vowel strength.

\section{References}

Adler, Jeff \& Jesse Zymet. (in press). Irreducible parallelism in phonology: evidence for lookahead from Mohawk, Maragoli, Sino-Japanese, and Lithuanian. Natural Language and Linguistic Theory.

Alderete, John. 1999. Head dependence in stress-epenthesis interaction. In Ben Hermans \& Marc van Oostendorp (eds.), The derivational residue in phonological Optimality Theory. 29-50. Amsterdam: John Benjamins.

Bellik, Jennifer. 2018. An acoustic study of vowel intrusion in Turkish onset clusters. Laboratory Phonology: Journal of the Association for Laboratory Phonology, 9(1), 16.

Cohn, Abigail \& John McCarthy. 1998. Alignment and parallelism in Indonesian phonology. Working papers of the Cornell Phonetics Laboratory 12.53-137.

Crowhurst, Megan \& Lev Michael. 2005. Iterative footing and prominence-driven stress in Nanti (Kampa). Language 81(1): 47-95.

De Lacy, Paul. 2004. Markedness conflation in Optimality Theory. Phonology 21(2). 145- 199.

Elfner, Emily. 2016. Stress-Epenthesis Interactions in Harmonic Serialism. In Harmonic Grammar and Harmonic Serialism, eds. John J. McCarthy and Joe Pater, 261-300. Sheffield: Equinox Press.

Hall, Nancy E. 2006. Cross-linguistic patterns of vowel intrusion. Journal of Phonology 23(3), 387-429.

Hammond, Michael, Natasha Warner, Andréa Davis, Andrew Carnie, Diana Archangeli, \& Muriel Fisher. 2014. Vowel insertion in Scottish Gaelic. Phonology 31. 123-153.

Hayes, Bruce. 1989. Compensatory lengthening in moraic phonology. Linguistic Inquiry 20.

Hyman, Larry M. 1985. A theory of phonological weight. Dordrecht: Foris.

Gafos, Adamantios I.. 2002. A grammar of gestural coordination. NLLT, 20(2), 269-337.

Garvin, Karee, Myriam Lapierre, and Sharon Inkelas. 2018. A Q-Theoretic approach to distinctive subsegmental timing. Proceedings of the 2018 Linguistic Society of America.

Inkelas, Sharon \& Stephanie S Shih. 2017. Looking into segments. Proceedings of the 2016 Annual Meeting on Phonology, ed. by Karen Jesney, Charlie O'Hara, Caitlin Smith, and Rachel Walker. Washington, DC: Linguistic Society of America.

Lapierre, Myriam. 2019. A Phonological Analysis of Panãra. Ph.D. Qualifying Paper, University of California, Berkeley.

Legendre, Geraldine, Yoshiro Miyata, and Paul Smolensky. 1990. Harmonic Grammar - A formal multi-level connectionist theory of linguistic well-formedness: An application. In Proceedings of the Twelfth Annual Conference of the Cognitive Science Society, 884-891. Mahwah, NJ: Lawrence Erlbaum.

McCarthy, John J. and Prince, Alan. 1994. Prosodic morphology. A Handbook of Phonological Theory. 15.

McKendry, Inga. (2013). Tonal Association, Prominence and Prosodic Structure in South-Eastern Nochixtlan Mixtec. $\mathrm{Ph} . \mathrm{D}$. thesis, University of Edinburgh.

Michelson, Karin. 1988. A Comparative Study of Lake-Iroquian Accent. Kluwer, Dordrecht: Kluwer Academic Publishers.

Michelson, Karin. 1989. Invisibility: Vowels without a Timing Slot in Mohawk. In Theoretical Perspectives on Native American Languages, eds. Donna B. Gerdts and Karin Michelson, 38-69. State University of New York Press, Albany.

Morén, Bruce. 2000. The puzzle of Kashmiri stress. Phonology 17: 365-396. 
Ryan, Kevin. 2018. VV > VC > V for Stress: Coercion vs. Prominence. Linguistic Inquiry 51:1-16.

Schwarz, Martha, Myriam Lapierre, Karee Garvin, and Sharon Inkelas. 2019. Recent Advances in Q Theory: Segment Strength. Proceedings of the 2019 Linguistics Society of America meeting.

Shih, Stephanie S \& Sharon Inkelas. 2019. Autosegmental Aims in Surface Optimizing Phonology. Linguistic Inquiry 50.137-196.

Smith, Caitlyn. 2017. Harmony triggering as a contrastive property of segments. Proceedings of the 2016 Annual Meeting on Phonology.

Staubs, Robert, Michael Becker, Christopher Potts, Patrick Pratt, John McCarthy, and Joe Pater. 2010. OT-help 2.0 software package. Amherst, MA. https://people.umass.edu/othelp.

Steriade, Donca. 1990. Gestures and autosegments: Comments on Browman and Goldstein's paper. In John Kingston \& Mary E. Beckman. (eds.), Papers in Laboratory Phonology 1, 382-397.

Zec, Draga. 1988. Sonority constraints on prosodic structure. Stanford University PhD dissertation.

Zec, Draga. 1995. Sonority constraints on syllable structure. Phonology 12.

Zec, Draga. 1998. Coda constraints and conditions on moraic projection. Working papers of the Cornell Phonetics Laboratory 12.

Zimmermann, Eva. (2018). Being exceptional is being weak: Tonal exceptions in San Miguel el Grande Mixtec. Gallagher, Gillian, et al. (eds.), Proceedings of Annual Meeting on Phonology, Linguistics Society of America, http://dx.doi.org/10.3765/amp. 